\title{
Features of the building of three-dimensional models of agricultural parcels of land to assess the influence of the relief on the signal stability of cellular networks
}

\author{
Stepan Vershinin *, Natalia Berezina, Igor Mysishin ${ }^{1}$ and Yulia Mikhaylova \\ Orel state agrarian university named after N.V. Parakhin 69, General Rodin Str., Orel, Russia
}

\begin{abstract}
The article examines main aspects of building threedimensional (3d) models of agricultural parcels of land on the example of generation of digital models of the experimental farm fields. Features and characteristics of the $3 \mathrm{~d}$ field models building are given according to the results of the application of photogrammetry methods using a UAV (quadcopter) to obtain a heightmap of the investigated areas to compare it with the change in the density of the GSM signal coverage. The examples of digital terrain models (DTMs) obtained at different stages of the processing are given, the issues of using ready-made models for further analytical research on the example of a heightmap transformation for tracking and predicting negative processes, are considered.
\end{abstract}

\section{Introduction}

The work is aimed at ensuring the continual use of digital support methods for agricultural production, improving accuracy and efficiency in the production of agricultural work (at all stages of agricultural production) and by the operational and general control as well. Different devices and complexes for monitoring and control, based on the use of global positioning systems (for accurate positioning) and transmitters based on GSM signals (for data transmission, including differential corrections for satellite positioning systems GLONASS (Russia) and GPS (USA), data processing and control systems) are widely used in agriculture.

The use of cellular communication for the transmission of digital information from monitoring objects to a remote service increases the efficiency of satellite control and monitoring technology greatly. The system can be used to transmit all types of information, including voice and video communication.

In most cases, a GSM signal provided by a certain cellular operator is used for on-line monitoring of the state of the monitored objects.

The quality and continuity of GSM communication affects greatly the ability to perform technological operations. Strays in the radio channel are caused both by the signal distortions during its propagation and by the external sources influence.

*Corresponding author: styvver@yandex.ru 
The most difficult is to eliminate the interference of external sources. Theoretically, an increase in the bandwidth-duration product (the product of the effective signal duration and the effective value of the bandwidth) makes it possible to reduce the strays to an arbitrarily small level.

The main difficulty in building a GSM channel is associated with ensuring continual GSM/GPRS communication with the operator. Large area of the cultivated territories, their remoteness from the cellular operator's infrastructure, significant inequality of the relief, presence of natural obstacles to wave propagation, as well as weather conditions, prevent certain types of agricultural and transport equipment from staying inside the coverage area of the cellular operators.

As part of the study on the GSM signal coverage density of the agricultural lands of the Scientific and Educational Production Center "Integration", a number of fields with the most complicated altitude characteristics (terrain) were digitized to assess the influence of the relief factors on the GSM signal stability for the effective placement of self-organizing sensor networks.

The terrain, the location, the remoteness and long distance of production sites, the availability of infrastructure and forest shelter belts have a significant negative impact on the signal propagation of the cellular operators. The GPS signal coverage of the cultivated areas in the agricultural organization may be insufficient for the implementation of the tasks of the digital transformation of production. This is especially relevant for the digital experimental farms, which are the drivers for the implementation and development of new technological solutions.

The object of the work was to obtain a heightmap of the survey plots to compare it with the change in the density of the GSM signal coverage (it is necessary to transfer relevant information from the machinery and equipment working in the fields to the data processing center). For digitization, photogrammetry methods [1] with the use of a quadcopter to obtain aerial shorts of the parcels were applied. The height of the survey varied from 100 to 220 meters, the area of the studied fields was from 27 to 142 hectares.

\section{Material and methods}

Drone-UAV (unmanned aerial vehicle) "AgroflyQuadro 4/17" was used for shooting. Its type is quadcopter (multi-rotor, horizontal speed - over $15 \mathrm{~m} / \mathrm{s}$, vertical speed - over $5 \mathrm{~m} /$ $\mathrm{s}$, flight height approx. $1.5 \mathrm{~km}$, flight time - up to $30 \mathrm{~min}$ ).

The control is carried out with the LX2-Navigator program - a software module as part of the GIS "Neva". 


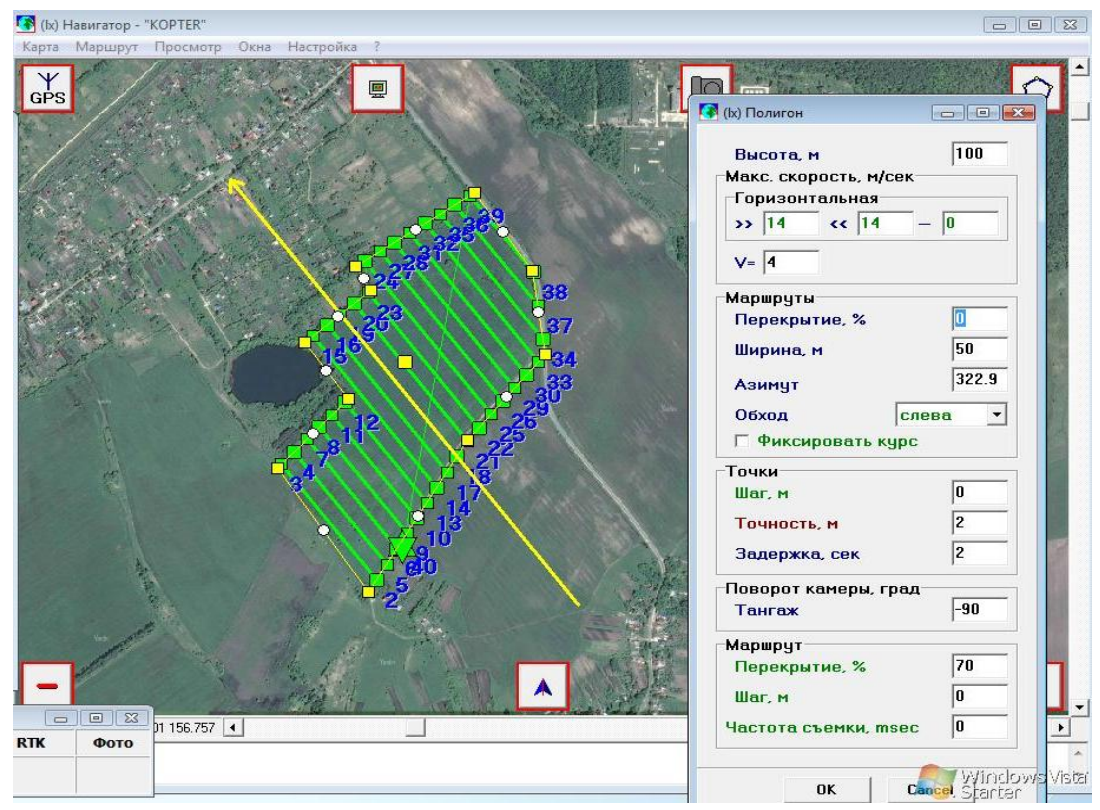

Fig. 1. An example of the flight task with the parameters.

The device is under a remote control via a radio module. The fulfillment of the flight task is automatic (autonomous), even if the communication with the control station (laptop) is lost. The images with the telemetry are saved to the internal memory of the device.

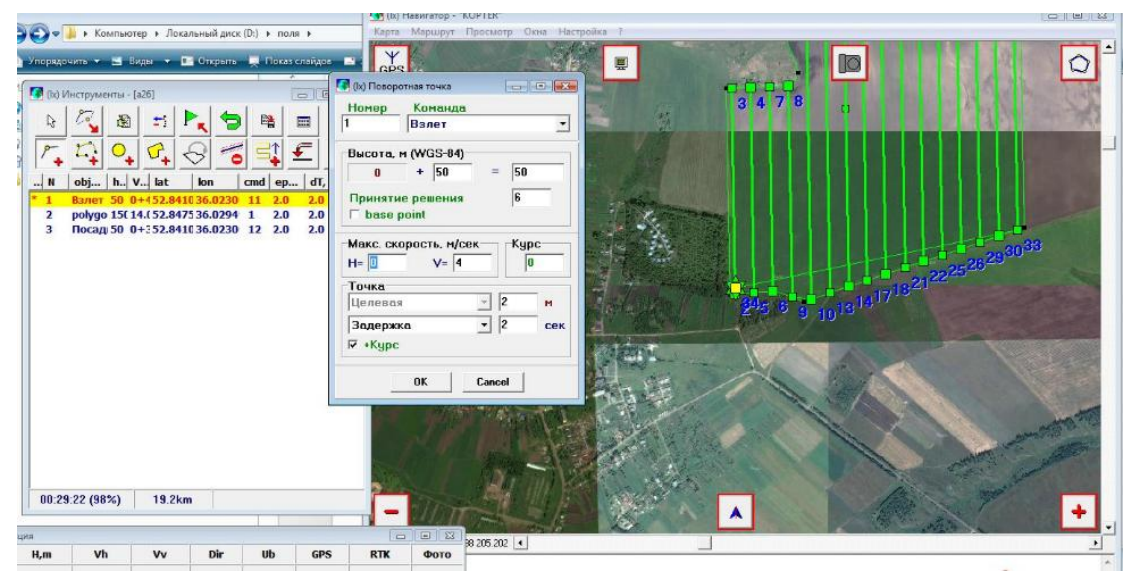

Fig. 2. The parameters of the take-off point and flight tasks.

The shooting is carried out by aerial photography, the number of trips (passes) depends on the height, overlap and area, the building of the terrain model is carried out using photogrammetry methods when processing in the Metashape-pro 1.5.1 program (creating a photo model, analyzing the relief, etc.).

Metashape consists of a set of measuring tools that can be used to calculate the distance between points, surface area and volume of the reconstructed $3 \mathrm{~d}$ model [2].

To reconstruct a $3 \mathrm{~d}$ model of the object, we used photographs taken with a digital camera of the quadcopter, each element of the reconstructed scene was captured with the overlap and was visible from at least two shooting positions) [3]. 
A set of GPS equipment was used for georeferencing, the referencing was carried out in the WGS 84 coordinate system (World Geodetic System 1984). Further data processing was carried out using the program Metashape. The main tasks that were solved using the Metashape program were $3 \mathrm{~d}$ landing reclamation and building of the orthophotomap and DTM (digital terrain model).

\section{Methodology for processing and analysing the data obtained with photogrammetry methods}

The work at the project was carried out (provided by the software package) in four stages:

At the initial stage, the parameters of external and internal orientation of the cameras were defined. The common points of the photographs (at least 2) were found and all the parameters of the cameras, such as general position of the cameras, orientation during shooting, the internal geometry of the images (focal length, distortion, etc.), were defined using them.

Based on the results of the calculations, a sparse point cloud was built in the $3 \mathrm{~d}$ space of the model and the data concerning position and orientation of the cameras were processed [4].

A sparse point cloud, as a rule, is not used at further stages of the processing (except for the mode of building a model based on a sparse point cloud) and it serves only for visual assessment of the photo alignment quality [2, p. v]. The sparse point cloud can be saved in various formats for the further use in other CAD software packages.

At the second stage, a dense point cloud is built. At the second stage, a dense point cloud was built based on the camera positions calculated at the first stage of the processing and the photographs used. To move to the next stage of creating a solid model or before exporting the model to intermediate formats, the dense point cloud must be edited and classified.

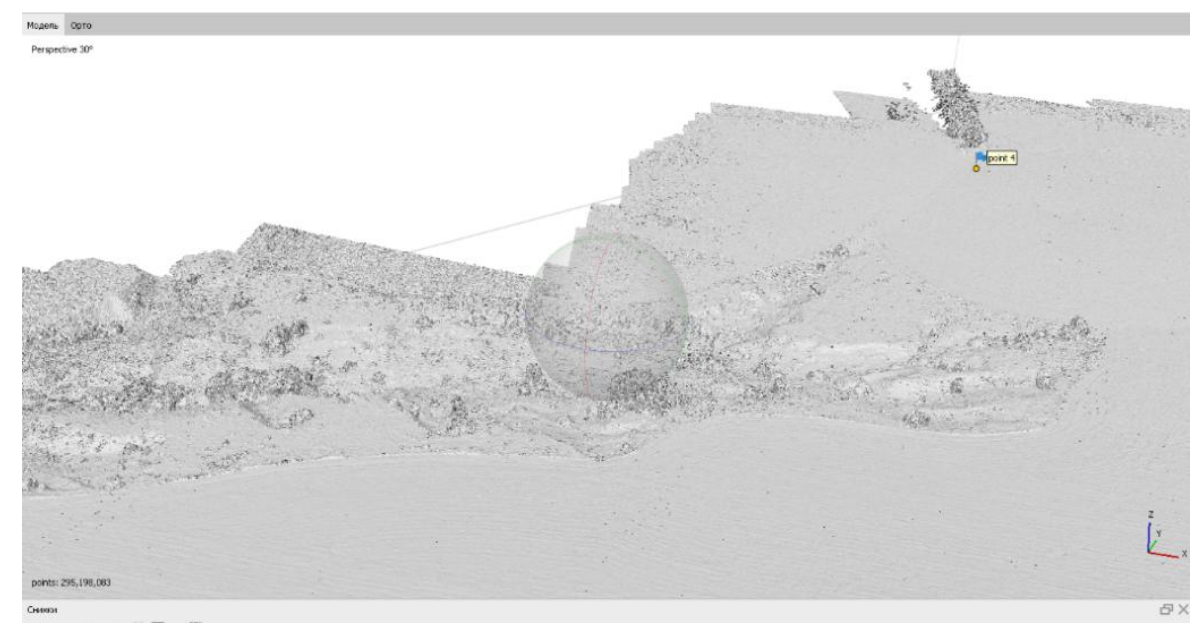

Fig. 3. Point cloud of the field parcel with an adjoining coombe (on the right).

A dense point cloud was created and displayed. Based on the calculated positions of the cameras, the program gives depth maps for each camera and builds a dense point cloud based on them [5]. At this stage, very dense point clouds are generated: they are similar to the point clouds in the aerial laser scanning in structure. Then the cloud data are to be edited and classified. It was done using the built-in Metashape functions, further the data were used to build a polygonal model, a heightmap and a tile model. 
At the third stage, modeling of a three-dimensional surface, a polygonal model and a heightmap was carried out. A 3d polygonal model describes a shape of the object based on the dense point cloud. It is also possible to build quickly a model based only on a sparse point cloud, but a dense point cloud was used for the most accurate calculation of the parameters. As a rule, a polygonal model is superfluous and it is possible to start building a heightmap immediately, abandoning the construction of the polygonal model, but for this project a complete construction methodology with the maximum visual representation was used [6].

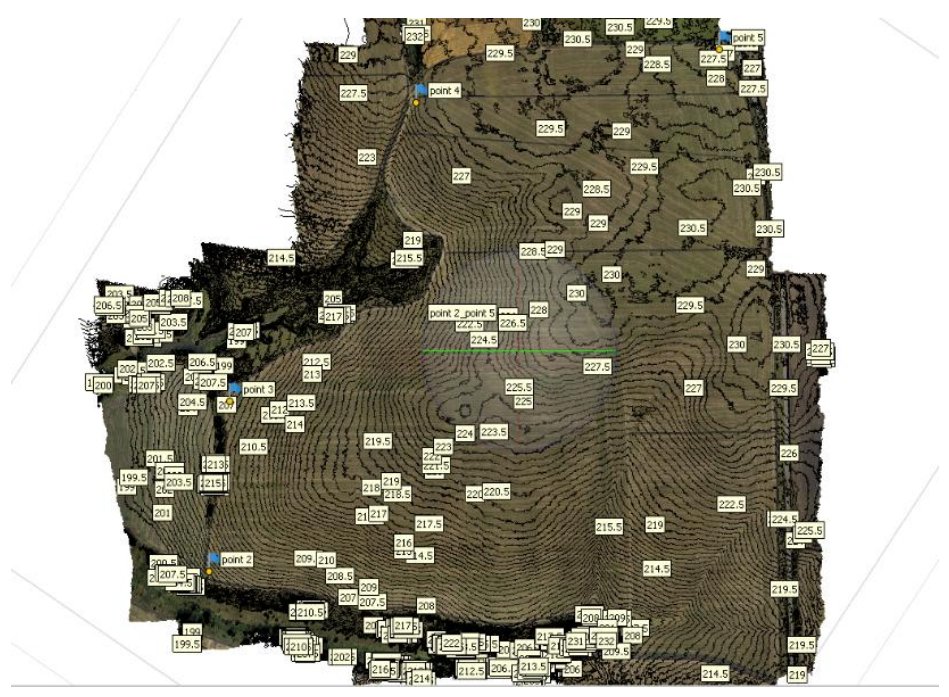

Fig. 4. The $3 \mathrm{~d}$ model of the parcel, maximum slope $5.8 \%$ (3.31 degrees), elevation changes 29 meters, max. height (WGS 84) $220 \mathrm{~m}$, min. $201 \mathrm{~m}$.

The determination and building of the texture for the polygonal model, as well as the construction of the orthophotomap are carried out at the last stage.

The orthophotomap was projected onto the surface in a geographic projection; the polygonal model was used for the basis of the orthophotomap construction.

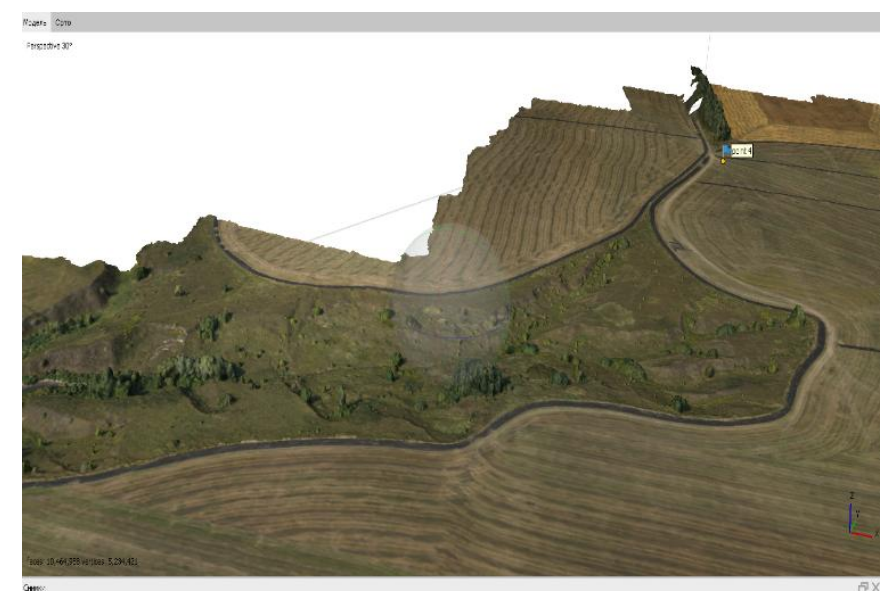

Fig. 5. Textured model of the field parcel. 
A 3d polygonal model based on a point cloud (dense) was reconstructed in the project.

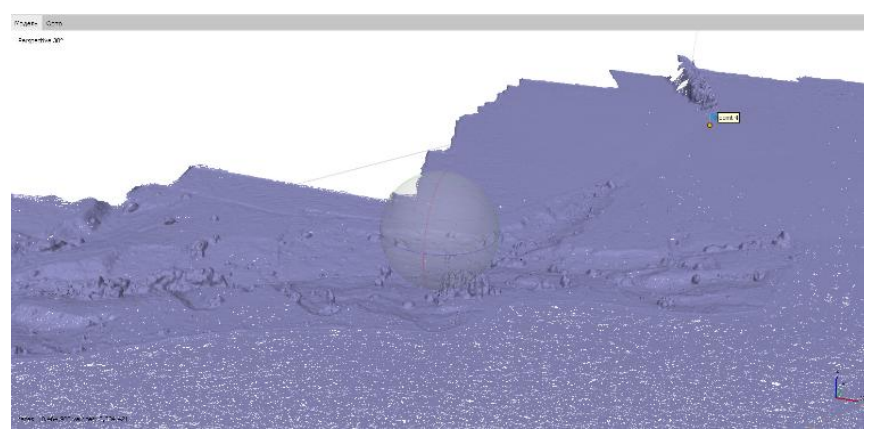

Fig. 6. Three-dimensional model of the field parcel.

It was mentioned above that in Metashape it is possible to use several methods for reconstructing a three-dimensional polygonal model, with the help of which one can perform optimal reconstruction for a specific set of photographs [2, p. 33].

1. Fast creation of the polygonal model based only on a sparse point cloud.

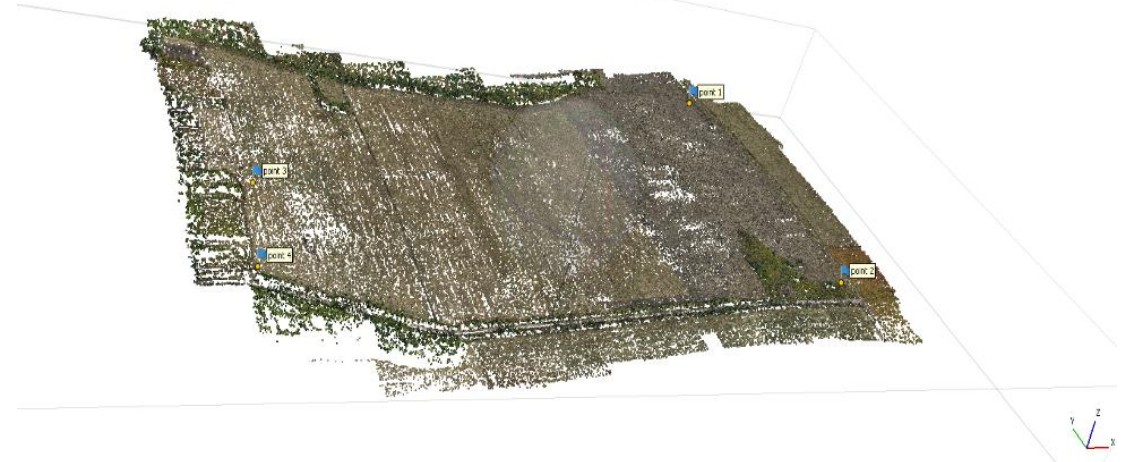

Fig. 7. Sparse point cloud with combined colors accordingto the mage textures.

2. Using a dense cloud it is possible to build a high-quality polygonal model, for it one needs a previously reconstructed dense point cloud. However, using this method, the processing time will increase significantly. For the assessment of the resulting polygonal model nature, a preliminary calculation was carried out using a sparse point cloud to eliminate possible errors at the stage of complete processing (to save the computing time resources), since if an error at the stage of calculations occurs, tens of hours of computer operations are lost. It is also possible to use the value of the depth map of the original photographs directly, which allows to use the information from the original images more efficiently, while, in comparison with building a model based on the dense cloud, it requires less resources and, as a result, less time for the calculations.

For modeling it is necessary to select a type of surface. The toolkit of the software package allows setting different base surfaces:

1. As a rule an arbitrary surface type is used to model objects of the arbitrary type. This type should be selected for the reconstruction of complex, irregular closed surfaces. There are no restrictions for the types of objects being modeled. 
2. The surface type "Heightmap" is optimized for modeling relatively flat surfaces, such as terrain. This type of base surface is accepted when processing the results of the aerial photography.

During the processing, the maximum number of polygons in the final three-dimensional polygonal model is defined [7]. Three values for the parameter are set: 1 . high; 2. average; 3 . low. They are calculated based on the number of points in the previously created dense cloud: the ratio is equal to $1 / 5,1 / 15$, and $1 / 45$ for the corresponding level [2, p. 34]. For this project it is optimal to use the "average" value of the parameter for the appropriate refinement.

Then the orthophotomap was built. The orthophotomap is built on the basis of the original photographs (it allows forming a final high-resolution image) and the reconstructed model [8]. The orthophotomap is, in fact, an end result of the aerial photography of the land surface.

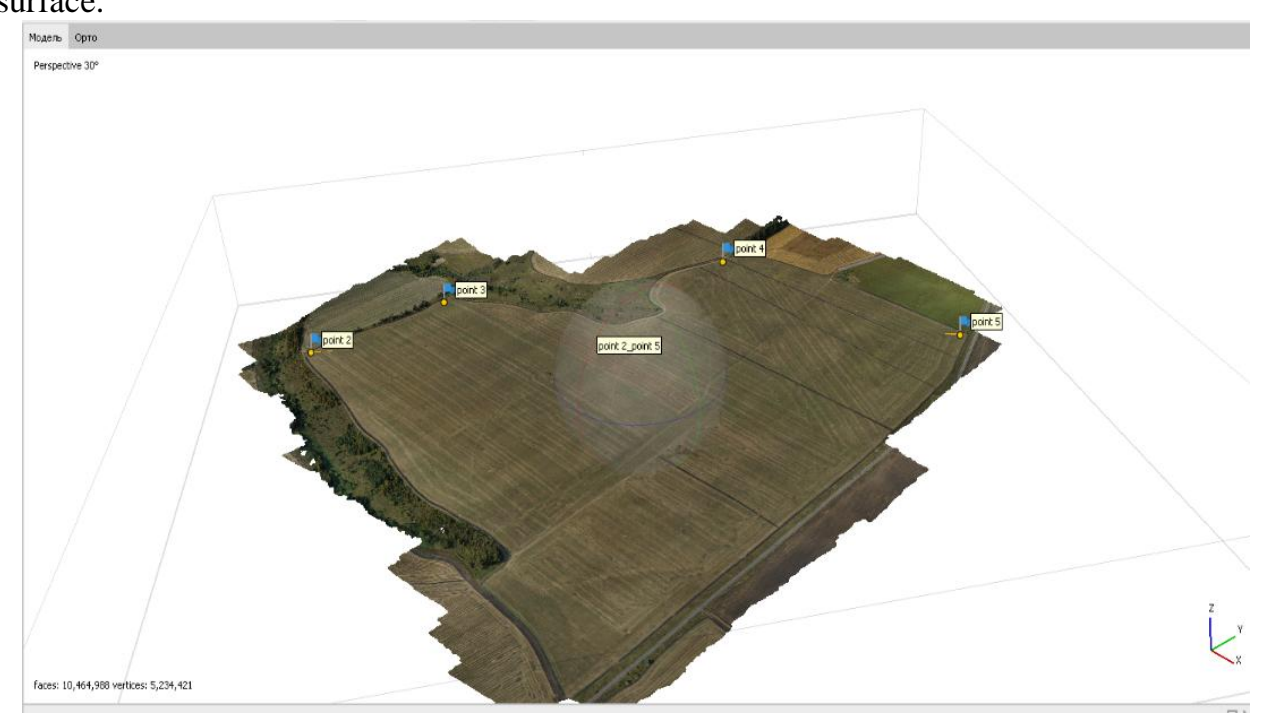

Fig. 8. 3d model of the parcel.

When building the orthophotomap, a geographic projection was used, the basis of which was a geographic coordinate system. User parameters of the geographic coordinate system were loaded. The projection was chosen relative to the coordinate axes, based on the current model orientation.

For accurate coordinates binding at least 4 markers per each parcel (not lying in one line) were used. For one of the parcels, it turned out to be impossible to use one of the 5 markers for the binding (it was in one of the corners, the picture was taken at the end of the flight task and there was a failure at that moment), which led to a decrease in the accuracy of the height positioning, but the presence of four other control markers allowed to bring the binding accuracy to the sufficient for the project level [9]. Therefore, it is preferable to use at least 5 control points. 


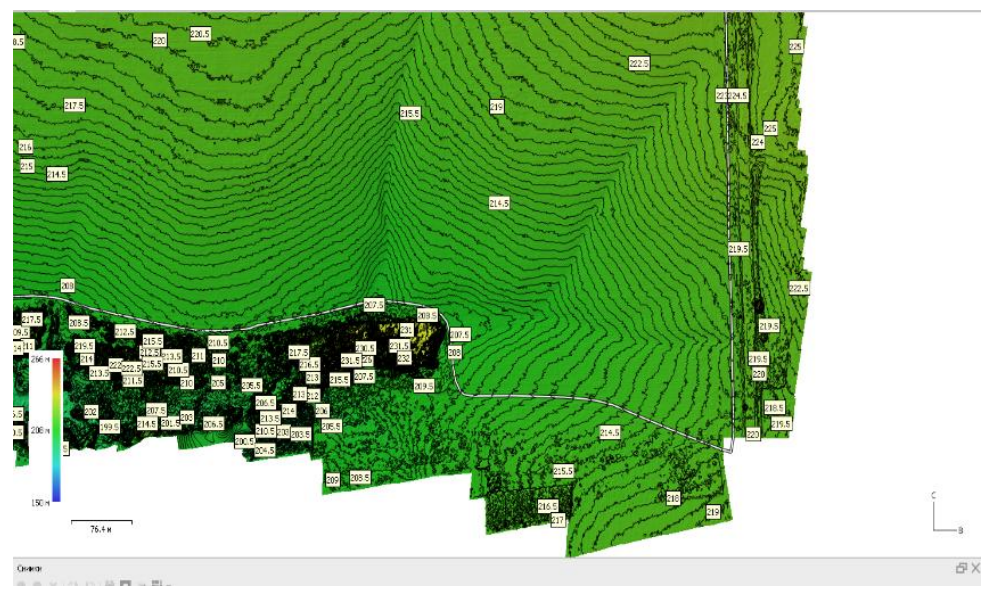

Fig. 9. DTM of the parcel with the development of linear erosion, a vector layer of contours is superimposed.

The main parameter for bitmap images (on the related textures of the $3 \mathrm{~d}$ model and connected with it digital models) is the pixel size value, this size corresponds to the average effective shot resolution. If one chooses a pixel size less than the effective average one, the size of the orthophotomap will increase (by increasing the number of pixels and density respectively), but not its effective resolution (since additional pixels are formed based on the results of interpolation of the initial pixels of the photography image), so the overall quality does not increase with an increase in the amount of the processed information $[10,11,12]$. The total orthophotomap resolution was $4.2-8.4 \mathrm{~cm} /$ pixel, which was set when processing the corresponding parcels.

When analyzing the relief of the fields, the toolkit to display the profile and contour lines was used. Metashape allows to monitor profiles along the lines defined using shapes (polylines and polygons). The sectional drawing is constructed along the specified route in the parallel surface. The profile for a polyline / polygon is calculated for all lines that make up the shape, starting with the first one drawn.

When comparing the created models of the farmland with the radio signal density map, it can be noted that it is not the absolute height of the field surface itself that mainly effects the quality of the GSM signal, but it is done by the sudden changes in the relief in the certain local areas, the strays are created both in the "negative" (hollows, coombes, etc.) and on the "positive" changes. But in the areas with elevation changes, the strays are much greater, especially when there is large difference in altitude $(10-15 \mathrm{~m})$ and the gradient is more than $12 \%$.

The analysis of the terrain profile was carried out according to the given lines.

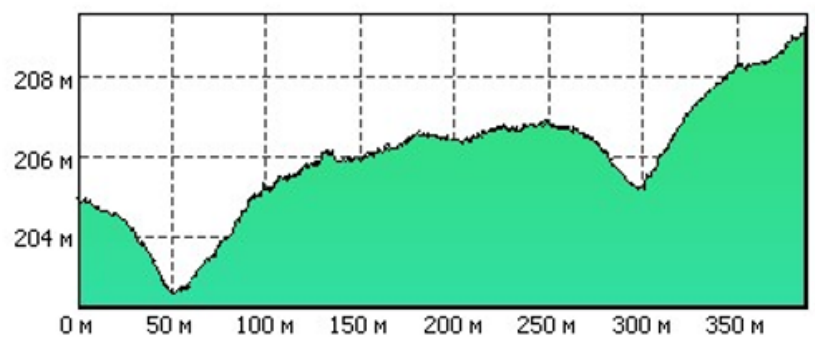

Fig. 10. An example of the profile built along the line (see Fig. 12). 
Initially, the sectional trace is set with a polyline or a polygon, then the calculation is performed in the given directions, the information is issued both in the form of horizontal and altitude coordinates of the control points, and in the form of a graphical representation (profile). Moreover, the scales along the horizontal and vertical axes change dynamically (for a more vivid visual representation of the height difference) and differ from each other (as a rule, the vertical and horizontal scales of the constructed profiles differ by 10 times).

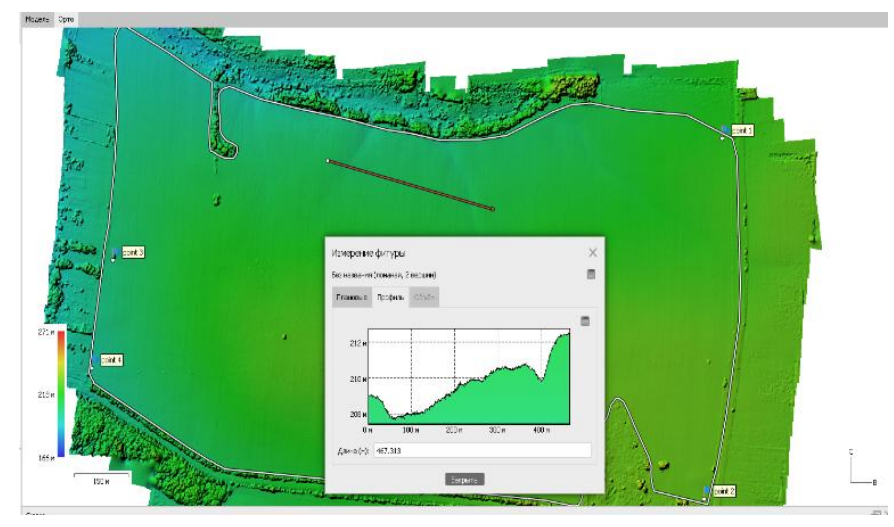

Fig. 11. DTM with a constructed profile along the givenline, maximum slope 4\% (2.29 degrees).

For further processing the export of the contour lines was used. It should be noted that there are limitations to this software package: a file with the SHP name suffix can include lines of only one type: either polylines or polygons (they are exported separately).

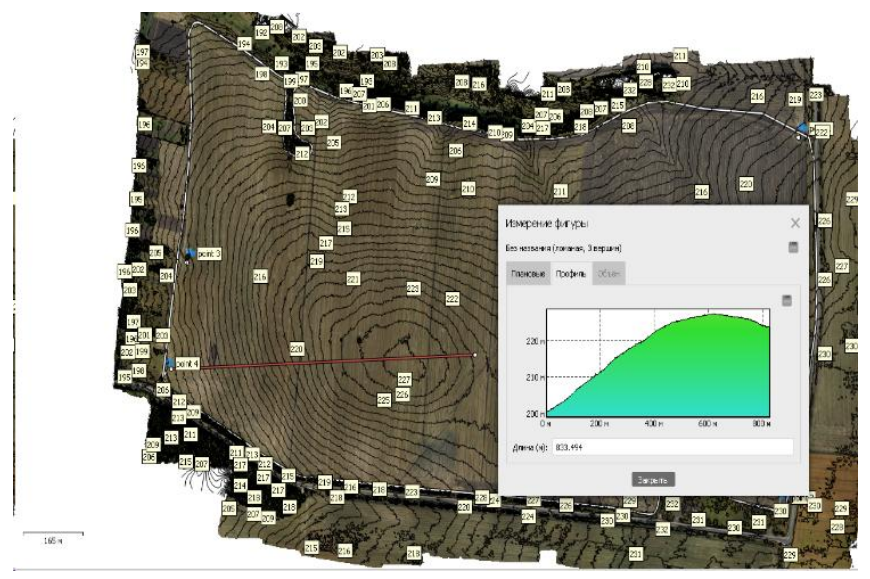

Fig. 12. Orthophotomap with a constructed profile alongthe given line, the maximum slope is $5 \%$ (2.86 degrees), the height difference is 26 meters.

Three areas with the development of linear erosion are clearly distinguished; a transition to the revinement and soil erosion processes is possible. The slopes are up to $6 \%$. 


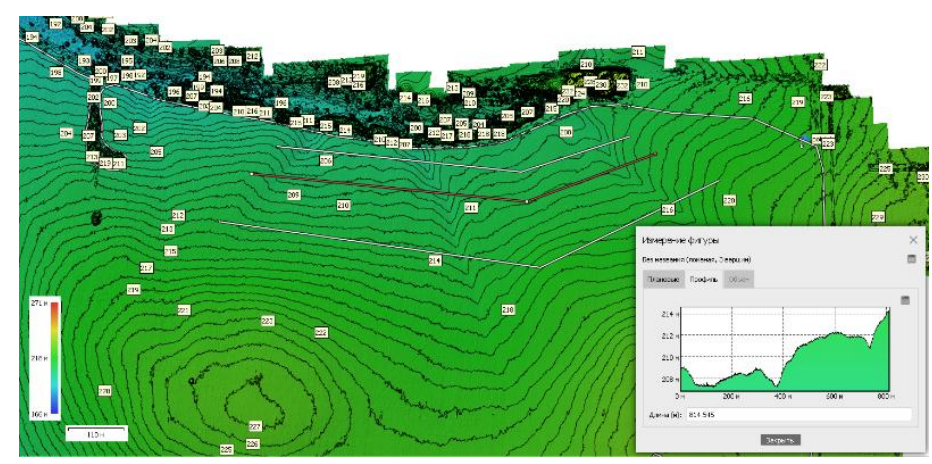

Fig. 13. DTM, area with the development of linear erosion with a constructed profile along the dedicated line 2.

\section{Conclusions}

The amount of the work done at this stage provides the basis for further observations. For subsequent studies, the heightmap conversion function can be used, allowing to calculate the difference between heightmaps for different periods of shooting and monitor changes to identify, predict and prevent negative processes, which will be used at the next stage of work. Secondary and subsequent monitoring of the objects can be performed only with the help of the aerial photography, without the use of land surveying equipment, which will reduce significantly the volume of both field work and calculations when processing data in the software systems. The photogrammetric method is a good alternative to laser scanning in the individual areas with the requirements for the scales accuracy 1: 1000, 1: 2000 and 1: 500. It should be noted that when photographic methods are used, there is no measurement redundancy, the obtained data do not need to be reduced.

The work was performed within the framework of the thematic plan-task for the research work commissioned by the Ministry of Agriculture of Russia at the expense of the federal budget in 2020 (registration number R\&D AAAA-A20-120021190096-3, dated 11.02.2020).

\section{References}

1. Anders N., Masselink R., Keesstra S., Suomalainen J., High-res digital surface modeling using fixed-wing UAV-based photogrammetry, Proceedings of the Geomorphometry (Nanjing China), 16-20 (2013)

2. AgisoftMetashape User Guide: Professional Edition, version 1.5 (Electronic resource) Agisoft LLC., 82 (2019)

3. Novel S., Keriven R., Grandorzh F., Pu F. Comparison of the aerial photogrammetry and three-dimensional laser scanning methods for creating three-dimensional models of the complex objects CADmaster: electron. journal 2 (84), 102-106 (2016)

4. Steve T., Generating 3D landscapes from Aerial Photos Part 2 Ibareitall, 230 (2015)

5. Remondino F., Remote Sensing, 3(6), 1104-1138 (2011)

6. López J.B., Jiménez G.A., Romero M.S., García E.A., Martín S.F., Medina A.L., Guerrero J.E., Journal of Archaeological Science, 10, 495-506 (2016)

7. El-Hakim S.F., Beraldin J.A, Picard M. and Godin G., Detailed 3D reconstruction of large-scale heritage sites with integrated techniques Computer Graphics and Applications IEEE 24(3), 21-29 (2004) 
8. Cawood A.J., Bond C.E., Howell J.A., Butler R. W H., Totake Y., LiDAR, Journal. Struct. Geol., 98, 67-82 (2017)

9. Remondino F., El-Hakim S., Image-based 3D Modeling: a Review The Photogrammetric, Record, Issue 21, 269-291 (2006)

10. Yin X., Wonka P., Razdan A., Generating 3D building models from architectural drawings, IEEE Comput., Graph., Appl. 29, 20-30 (2009)

11. Faisal M., Khizar A., Asif R., Muhammad A.K., Prince Waqas K., , International Journal of Advanced Computer Science and Applications. 10, 1, 443-449 (2019)

12. M.A. Nikitina, I.A. Nikitin, N.G. Semenkina, I.V. Zavalishin, A.V. Goncharov, International Journal of Advanced Computer Science and Applications. 9 (5), 51-59 (2018) 